\title{
Tetrathiotetracene thin film morphology and electrical properties
}

\author{
K. Pudzs ${ }^{1 *}$, A. Vembris ${ }^{1}$, J. Busenbergs ${ }^{1}$, M. Rutkis ${ }^{1}$ and \\ S. Woodward ${ }^{2}$ \\ ${ }^{1}$ Institute of Solid State Physics, University of Latvia, 8 Kengaraga \\ Street, Riga LV-1063, Latvia \\ ${ }^{2}$ School of Chemistry, The University of Nottingham, University Park, \\ Nottingham, NG7 2RD, United Kingdom \\ E-mail: kaspars.pudzs@cfi.lu.lv, Fax: +37167132778, Tel: \\ $+37167260787$
}

\begin{abstract}
The electrical properties of organic thin films are determined by their chemical constituents and the morphology of the films deposited. In this paper the morphology of vacuum sublimed $\left(7 \cdot 10^{-6} \mathrm{mbar}\right)$ tetrathiotetracene (TTT) thin films is shown to be strongly affected by the thermal deposition temperature $(222-350 \mathrm{~K})$ and rate of deposition. Mostly needle-like morphologies are identified by scanning electron microscopy. Optimal TTT purity (a pre-requisite for device preparation via subsequent oxidation) is evidenced by their initially low electrical conductivity. Altering the TTT morphology, by variation of the evaporation parameters, strongly affects this base electrical conductivity. Four probe conductivity measurements and charge extraction by linear increasing voltage methods are used to characterize film electrical properties. In-plane conductivity of up to $7.03 \cdot 10^{-5}$ $\mathrm{S} / \mathrm{cm}$ is achieved for pure TTT thin films. Subsequent aerial oxidation resulted in a 3.4-fold increase in electrical conductivity.
\end{abstract}

Keywords: tetrathiotetracene; thin film; electrical conductivity; morphology 


\section{Introduction}

Thermoelectric (TE) devices which can provide direct thermal to electrical energy conversion of low temperature waste heat $\left(<200{ }^{\circ} \mathrm{C}\right)$ could potentially help reduce global warming if their widespread utilisation could be realized. Presently the materials used for efficient TE devices are expensive, often not widely available and normally contain unsustainable or toxic elements such as $\mathrm{Te}$ and $\mathrm{Pb}$ [1-5]. Organic materials with good TE properties could be a solution to these issues. High-electrical conductivity materials are a prerequisite for TE generators. One class of candidates for TE devices are radical cation salts attained by oxidation of tetrathiotetracene (TTT, Figure 1) [6-8], such as ditetrathiotetracene triiodide [9]. The TE performance of these salts is often compromised if non pristine TTT starting material is used and (low) electrical conductivity of the initial TTT is frequently the best indicator of its purity [9].

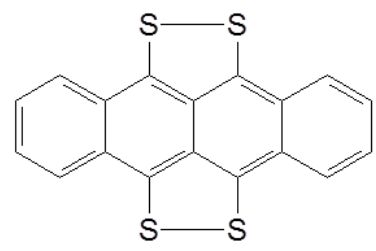

Figure 1. Chemical structure of tetrathiotetracene (TTT).

Large, good quality organic crystals with low impurity/defect concentrations often have higher electrical conductivity than polycrystalline films, but are challenging to grow. It is much easier to produce thin films of organic materials by thermal evaporation in vacuum techniques, but typically such films are polycrystalline. Consequently, it is necessary to investigate the impact of deposition technological parameters on the morphology and electrical properties of such films and TTT was selected for the present study. During (or after) evaporation TTT thin films can be doped with oxidants, such as iodide, dramatically increasing their conductivity. The electrical properties of such doped thin films may therefore depend on the initial TTT thin film morphology. For example, related morphology changes in polycrystalline pentacene films and their effect on electrical properties has been widely studied [10-12]. Some electrical properties of vacuum deposited TTT films have been investigated a long time ago [13-16]. For example, Inokuchi et al. reported TTT electrical resistivity reaching $1.3 \cdot 10^{6} \Omega \cdot \mathrm{cm}$ for thin films and $8.5 \cdot 10^{3} \Omega \cdot \mathrm{cm}$ for compressed pellets [13]. Webb et al. through DC and AC conduction studies attained a density of states of $\sim 10^{19} \mathrm{~cm}^{-3}$ for TTT [14] with thermally activated DC transport with an activation energy of $0.28 \mathrm{eV}$. The latter is very close to the $0.3 \mathrm{eV}$ trap level Balode et al. reported for TTT thin films [16]. To the best of our knowledge no investigation into how the morphology of TTT thin films affects 
electrical conductivity has been described. Recently, Fiedler et al. studied the structure of TTT monolayers on gold [17]. They found two different TTT monolayer phases on gold (111) surfaces depending on the amounts of TTT deposited. At low TTT concentrations on surface a disordered phase is seen which converts to an ordered phase at higher TTT surface coverage (the latter was assigned to 'flat' $\pi$-adsorption of TTT, the former containing additional 'edgeon' Au-S contacts). In this paper we report the influence of TTT deposition process parameters, including evaporation rate and substrate temperature, on the morphology and electrical properties of resultant polycrystalline TTT thin films of $650 \mathrm{~nm}$ to $2.5 \mu \mathrm{m}$ thickness.

\section{Experimental details}

\subsection{Tetrathiotetracene (TTT)}

The tetrathiotetracene (TTT, Figure 1) used in these experiments was synthesized in Bulgarian Academy of Sciences by Prof. Dr. V. Dimitrov and co-workers by a literature method [18] and purified several times by thermal gradient sublimation at the University of Wurzburg by the group of Prof. Dr. J. Pflaum. On the basis of ${ }^{1} \mathrm{H}$ NMR spectroscopy [400 MHz, ca. $0.3 \mathrm{mM}$ solutions in $\mathrm{d}_{8}$-toluene, $\left.\delta_{\mathrm{H}} 7.23-7.21(\mathrm{~m}, 4 \mathrm{H}), 6.96-6.90(\mathrm{~m}, 4 \mathrm{H})\right]$ and mass spectrometry the initial material was $>95 \%$ pure and the sublimed material $>98 \%$ TTT. Further enrichment can be expected during film deposition.

\subsection{Sample preparation}

A specially designed sample configuration (Figure 2) allows for the measurement of electrical conductivity both laterally (in-plane) and normal to the film's surface. For measurements of thin film in-plane conductivity we used four gold electrodes placed under the TTT layer. Basal (ITO) and top deposited ( $\mathrm{Al}$ ) electrodes were used to carry out measurements perpendicular to the TTT thin films. Sample preparation began by applying Scotch Crystal tape as mask to define the desired shape of the ITO electrode. Surplus ITO was etched away from the 25 by $25 \mathrm{~mm}$ glass slide using hydrochloride acid (concentration $10 \mathrm{M}$ ) and zinc pellets. The zinc reacts with hydrochloride acid producing hydrogen which together with the hydrochloride acid etches ITO. After removing the tape mask, the substrate was cleaned in turn with deionized water, acetone, chloroform and Helmanex III detergent dilution 2\%, more deionized water and finally isopropanol using $15 \mathrm{~min}$ cycles in an ultrasonic bath. After cleaning the sample was fixed on a special sample holder and loaded into the vacuum

chamber. Four gold electrodes were deposited by thermal evaporation in vacuum $\left(7 \cdot 10^{-6}\right.$ mbar) using shadow masks. Various TTT thin films were then deposited by thermal 
evaporation under vacuum by altering the substrate temperatures and TTT evaporation rates. Substrate temperatures in the range 222-350 K, and TTT mass evaporation rates between 45 $\mathrm{ng} \cdot \mathrm{cm}^{-2} \cdot \mathrm{s}^{-1}$ and $363 \mathrm{ng} \cdot \mathrm{cm}^{-2} \cdot \mathrm{s}^{-1}$ were used. Finally, the top Al electrodes were deposited the same way as gold under the same pumping cycle. Substrate temperature was controlled by a Peltier element built into the sample holder. Evaporation rates were monitored by in house fabricated quartz crystal resonators. The base pressure in the vacuum chamber was $7 \cdot 10^{-6}$ mbar. The actual thickness of the TTT films obtained was measured with profilometer Dektak 150. In all runs the same amount of TTT was used $(20 \mathrm{mg})$, but as measured by the profilometer, the film thickness varied in range of $650 \mathrm{~nm}$ to $2.5 \mu \mathrm{m}$ depending on the deposition parameters and the resultant film morphology.

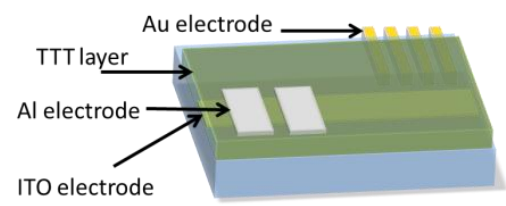

Figure 2. Schematic representation of the sample. Four Au electrodes under the TTT films were used for measurements of thin film conductivity in-plane. Bottom ITO and top Al electrodes were used to carry out conductivity measurements perpendicular to the TTT thin film.

\subsection{Experimental setups}

Electrical conductivity in the plane of film was measured by a four probe technique using the Au electrodes. A Keithley 6487 picoampermeter was used as the current source and voltage measurements were carried out with a Keithley 6514 instrument. The length of Au electrodes was $6 \mathrm{~mm}$ and the distance between them was $2 \mathrm{~mm}$; I-V curves with ohmic behaviour were obtained.

Charge carrier mobility and electrical conductivity perpendicular to the plane were measured using the Charge Extraction by Linear Increasing Voltage (CELIV) method [19,20]. Current transients were measured with a Tektronix DPO 2012 oscilloscope. Linear increasing voltages (up to $5 \mathrm{~V}$ with increasing at $5 \cdot 10^{3}$ to $1 \cdot 10^{6} \mathrm{~V} / \mathrm{s}$ ) were applied to the sample by a Tektronix AFG3021B arbitrary function generator.

Both in-plane and perpendicular to the plane electrical measurements were performed in ambient temperatures $\left(\mathrm{ca} .20^{\circ} \mathrm{C}\right)$.

Thin film morphology was studied by scanning electron microscopy using a Tescan Lyra 3 FEF-SEM $\times$ FIB with a $5 \mathrm{keV}$ electron beam. The conductivity of the TTT layers were high 
enough to avoid surface charging and therefore additional sputtering of the thin film with a conductive metal layer was not necessary.

\section{Results and Discussion}

Our sample configuration (Figure 2) allows us to characterize TTT morphology on different substrates - bare glass, ITO and gold covered glass were selected for the present study. The effect of substrate type was compared using identical deposition conditions. The morphologies of the TTT films deposited with an evaporation rate of $45 \mathrm{ng} \cdot \mathrm{cm}^{-2} \cdot \mathrm{s}^{-1}$ on the three substrates types above at three different temperatures $(350,300$ and $222 \mathrm{~K})$ are shown in Figure 3.
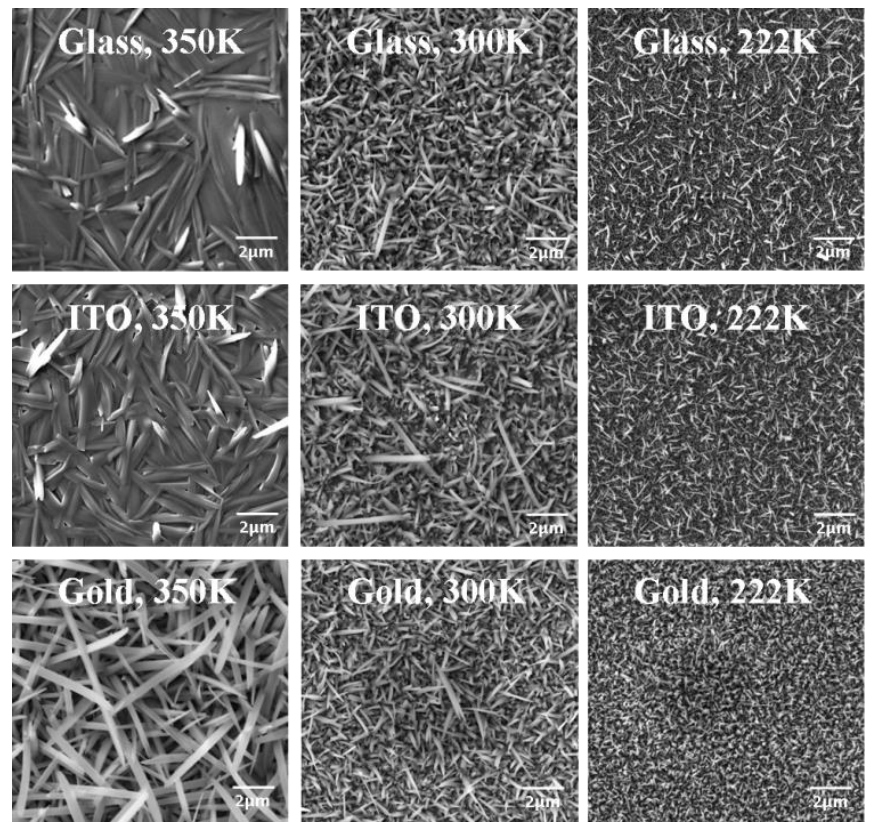

60102201

(1)

r.m.

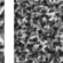

Whe

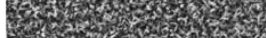

Figure 3. SEM images of the TTT thin films on different substrates at different temperatures during sublimation. Deposition rate $45 \mathrm{ng} \cdot \mathrm{cm}^{-2} \cdot \mathrm{s}^{-1}$.

A characteristic basal layer of small TTT grains was initially deposited on thin films grown on bare and ITO covered glass. Above this basal layer larger needle-shaped crystals grew. In case of ITO these needle-shape crystals were more pronounced. No base layers of smaller grains were seen for TTT films grown on bottom gold electrodes - needle-shaped crystals deposited spontaneously. Fiedler et al. has shown that on gold (111) surfaces TTT lies flat (planar orientation) and forms ordered structures above $270 \mathrm{~K}$ [17]. As amorphous (evaporated) gold thin films are used in our experiments the initial orientation of crystals is not expected to be very pronounced.

As expected, temperature influences the size of the crystals on all types of substrates. In thin films deposited at low substrate temperature the crystallites are smaller and consequently can 
pack more densely than those deposited at higher temperatures (Figure 3). Further evidence for more dense packing at lower temperature is provided by thin film thickness. Films deposited at lower temperature substrates were thinner than those deposited at higher temperatures. On gold substrates using the same amount of TTT $(20 \mathrm{mg})$ and evaporation rate $\left(45 \mathrm{ng} / \mathrm{cm}^{2} \mathrm{~s}\right)$ the thicknesses of obtained films at different temperatures were: $2.50 \mu \mathrm{m}$ (350 $\mathrm{K}), 1.40 \mu \mathrm{m}(300 \mathrm{~K})$ and $0.88 \mu \mathrm{m}(222 \mathrm{~K})$. At higher substrate temperatures different types of crystal packing occur which are substrate dependant. On gold the TTT crystals show a wide angular distribution but are more vertically oriented, whereas those crystals on ITO and glass are oriented largely in the thin film plane (Figure 3). Due to the different crystallite packaging one could expect different electrical properties of the TTT thin films.

In-plane electrical conductivity measurements were made during film deposition process. For the sample deposited on glass at a substrate temperature of $222 \mathrm{~K}$ the electrical conductivity dependence on the mass of deposited TTT is shown in Figure 4. The deposited mass is used (rather than film thickness) as the quartz crystal oscillator measures the former accurately, while the latter cannot be determined during the run and is morphology dependant. As one can see from the logarithmic inset, the conductivity increases exponentially up to $33 \mu \mathrm{g} / \mathrm{cm}^{2}$. One explanation for this is that this initial conductivity does not describe bulk material. If the initial deposition is not uniform but consists of small clusters of TTT forming a percolation network, the conductivity between the neighbouring clusters will be defined by tunnelling mechanism. After $33 \mu \mathrm{g} / \mathrm{cm}^{2}$ of TTT have been deposited film growth becomes linear and describes bulk material conductivity changes with growing cross-section of the layer. We estimate that this onset corresponds to film thicknesses of $\sim 300 \mathrm{~nm}$, but this value is rather dependant on the film morphology - crystal size, crystal orientation and packing density.

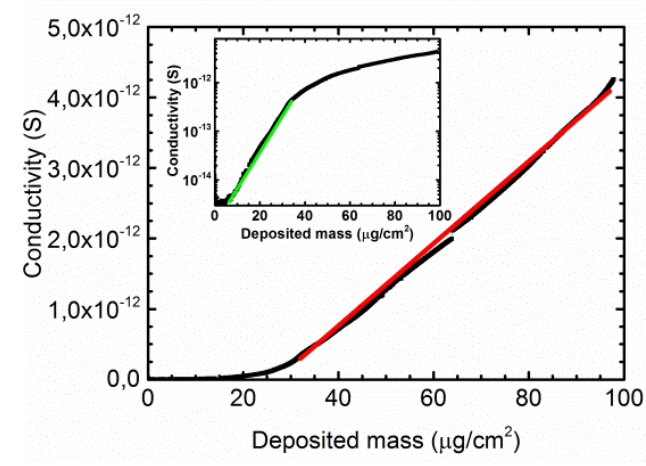

Figure 4. Linear and exponential electrical conductivity dependence on TTT thin film thickness Deposited with rate of $45 \mathrm{ng} / \mathrm{cm}^{2} \mathrm{~s}$ on glass at $222 \mathrm{~K}$.

The electrical properties measured under ambient laboratory conditions in air at room temperature for a representative TTT film sample are summarized in Table 1. Charge carrier 
mobility and conductivity perpendicular to the plane for the sample prepared at a substrate temperature of $350 \mathrm{~K}$ could not be determined. The TTT crystal spacing was so large (Figure 3) that attempted deposition of the top ( $\mathrm{Al})$ electrode lead only to short circuiting through penetration to the basal electrode. In the case of needle-shaped organic crystals electrical conductivity measured longitudinally can be several orders of magnitude higher than transversal conductivity [9]. Hilti et al. reported the anisotropy of conductivity in (TTT) ${ }_{2} \mathrm{I}_{3}$ crystals is between 100 and 1000 [21]. Our highest in-plane conductivity is for thin film, made at substrate temperatures of $350 \mathrm{~K}$ on glass (Table 1). Only in this case most of the crystals are placed with their long axes strongly orientated in the plane of thin film, however not in one dominant direction (Figure 3, glass and $350 \mathrm{~K}$ ). Nevertheless, charge carriers moving laterally in such films could use easy pass along the crystals supported by carrier hopping from one crystal to another. At lower substrate temperatures in-plane conductivity decreases with the crystal size (Table 1). The highest conductivity and charge carrier mobility perpendicular to the thin film plane is for the sample made at lowest (222 K) substrate temperature where the crystals are the smallest with mixed orientations. This means that we cannot deconvolute the specific in-plane and perpendicular contributions to the electrical conductivity for the same sample. For crystal anisotropic reasons in-plane orientation of crystals is optimal for achieving high lateral conductivity. For the same samples the conductivity perpendicular to the film plane is two orders of magnitude lower despite the fact that the charge carrier pathway through the crystal is short (Table 1). Unfortunately, in these randomly oriented small crystals the charge carrier mobility for such polycrystalline films is limited by the grain boundaries. In the case of closer packing, due to the smaller distance between them, the electron hopping and transport routes are smaller, closer and parallel to the surface.

Table 1. Electrical properties of the TTT thin films obtained at different substrate temperatures during sublimation. TTT deposited with rate of $45 \mathrm{ng} / \mathrm{cm}^{2} \mathrm{~s}$ on glass for in plane measurement and on ITO for perpendicular to the plane measurement.

\begin{tabular}{cccc}
\hline & $\mathrm{T}_{\mathrm{s}}=350 \mathrm{~K}$ & $\mathrm{~T}_{\mathrm{s}}=300 \mathrm{~K}$ & $\mathrm{~T}_{\mathrm{s}}=222 \mathrm{~K}$ \\
\hline$\sigma_{\text {planar }}, \mathrm{S} / \mathrm{cm}$ & $(2.67 \pm 0.21) \cdot 10^{-5}$ & $(2.37 \pm 0.21) \cdot 10^{-5}$ & $(1.75 \pm 0.21) \cdot 10^{-5}$ \\
$\sigma_{\text {normal }}, \mathrm{S} / \mathrm{cm}$ & - & $1.21 \cdot 10^{-7}$ & $1.76 \cdot 10^{-6}$ \\
$\mu_{\text {normal }}, \mathrm{cm}^{2} / \mathrm{V} \mathrm{s}$ & - & $1.19 \cdot 10^{-3}$ & $1.9 \cdot 10^{-3}$ \\
\hline
\end{tabular}

Evaporation rate also has an impact on the morphology of TTT thin films grown on bare glass substrates at temperature $300 \mathrm{~K}$ as shown in Figure 5. The evaporation rate is determined by quartz crystal resonator frequency shift in time. As expected, films obtained at the lowest evaporation rate have more pronounced crystalline appearance. By increasing evaporation 
rate the size of crystallites in thin film decreases resulting in more dense packing. The inplane conductivity and thin film density is summarized in Table 2. The highest in-plane conductivity $7.03 \cdot 10^{-5} \mathrm{~S} / \mathrm{cm}$ is for the sample with the highest density obtained at evaporation rate $363 \mathrm{ng} / \mathrm{cm}^{2} \mathrm{~s}$ which is equivalent to $2.5 \mathrm{~nm} / \mathrm{s}$. In this case obtained thin film is with the smallest crystallites with the closest inter-crystal distances.
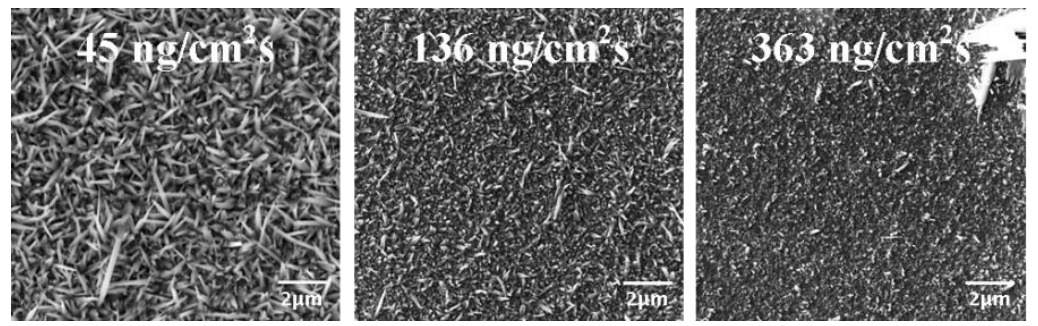

Figure 5. SEM images of the TTT thin films obtained at different evaporation rates on glass at $300 \mathrm{~K}$.

The conductivity dependence on crystallite size in Table 2 is opposite to the temperature dependence studies of Table 1. Both low temperatures and high initial TTT surface coverage favour molecular nucleation and this leads to the growth of dense films. The packing density has the dominant role in the electrical conductivity over crystallite size and orientation. Our electrical conductivity values for TTT thin films are higher than Inokuchi et al. who reported $7.69 \cdot 10^{-7} \mathrm{~S} / \mathrm{cm}$ for his TTT films [13]. Matsunaga reported even higher values of $10^{-4} \mathrm{~S} / \mathrm{cm}$ for TTT [22], but the sample preparation method and its type is unknown in this case.

Table 2. Density and electrical in-plane conductivity of TTT thin film obtained with different evaporation rates at substrate temperature $300 \mathrm{~K}$ on bare glass substrates.

\begin{tabular}{ccc}
\hline $\begin{array}{c}\text { Evaporation rate, } \\
\mathrm{ng} / \mathrm{cm}^{2} \mathrm{~s}\end{array}$ & $\begin{array}{c}\text { Thin film density, } \\
\mathrm{g} / \mathrm{cm}^{3}\end{array}$ & $\sigma_{\text {planar, }} \mathrm{S} / \mathrm{cm}$ \\
\hline 45 & 0.93 & $(2.37 \pm 0.21) \cdot 10^{-5}$ \\
136 & 1.22 & $(6.04 \pm 0.21) \cdot 10^{-5}$ \\
363 & 1.80 & $(7.03 \pm 0.21) \cdot 10^{-5}$ \\
\hline
\end{tabular}

Matsunaga suggested that low resistivity of his TTT samples resulted from oxidation and formation of TTT-oxygen species [22]. Inokuchi et al. [13] has reported a change of one order of magnitude in the electrical conductivity for TTT prepared in high vacuum and then exposed to oxygen. Similar conductivity changes were observed for our samples by four probe conductivity measurements made after admittance of air at the termination of our runs. In a representative example (Figure 6) the chamber was first re-pressurized with $\mathrm{N}_{2}$ to 1 bar 
and no changes in TTT film conductivity were observed. Subsequently the chamber was exposed to the air resulting in an increase of thin film conductivity by 3.4 times from $5.13 \cdot 10^{-6}$ to $1.75 \cdot 10^{-5} \mathrm{~S} / \mathrm{cm}$. In control experiments repeated evacuation of the air from chamber did not cause any further change or reversibility in the measured conductance. We conclude that irreversible oxidation of at least some of the TTT takes place but the resultant products could not be characterised.

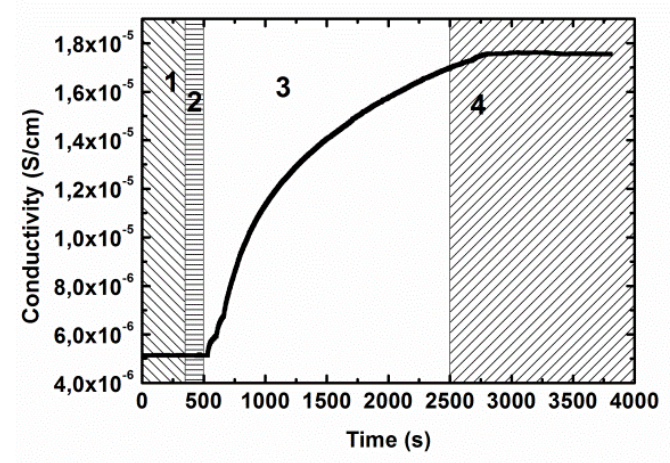

Figure 6. Air impact on TTT thin film conductivity. 1 - Vacuum, $6 \cdot 10^{-4} \mathrm{~Pa}$, turbo pump decelerating,

$\mathbf{2}-\mathrm{N}_{2}$ is inflated in the chamber, $\mathbf{3}$-Chamber is exposed to the air, $\mathbf{4}$-turbo molecular pump accelerating, vacuum. Sample grown on glass at $222 \mathrm{~K}$ and deposition rate $45 \mathrm{ng} / \mathrm{cm}^{2} \mathrm{~s}$.

\section{Conclusions}

Threshold amounts of deposited TTT $\left(>33 \mu \mathrm{g} / \mathrm{cm}^{2}\right)$ are necessary to attain bulk material which has the highest electrical conductivity. We suggest two charge carrier transport mechanisms: Charge carriers tunnelling between TTT clusters take place at the beginning of film growth due to the formation of percolation network. Hopping mechanism becomes dominant at certain film thickness of material where bulk material conductivity occurs. The conductivity in the TTT films is anisotropic and depends on crystal size, packing density and alignment. It can be partially moderated by appropriate control of substrate type and temperature and TTT deposition rate. Larger and more in-plane orientated crystals are favoured by increasing substrate temperature to $350 \mathrm{~K}$. This increases the in-plane contribution to the electrical conductivity at the expense of the perpendicular component. Higher packing density and smaller crystals are favoured by increasing TTT deposition rates and lower temperatures. The improved packing of smaller poorly ordered TTT crystals provides higher in-plane conductivity than films with higher proportions of in-plane orientated larger crystals. Packing density has the pivotal role in the conductivity rather than crystallite size. Oxidation of TTT films offers the potential to access materials with useful electrical conductivities and this will be reported in due course. 


\section{Acknowledgments}

This work has been supported by European Commission 7th Framework Programme project

"Waste Heat to Electrical Energy via Sustainable Organic Thermoelectric Devices". 


\section{References}

[1] K. Biswas, J. He, I.D. Blum, Chun-IWu, T.P. Hogan, D.N. Seidman, et al., Corrigendum: High-performance bulk thermoelectrics with all-scale hierarchical architectures, Nature. 490 (2012) 414-418.

[2] Z.H. Dughaish, Lead telluride as a thermoelectric material for thermoelectric power generation, Physica B Condens. Matter. 322 (2002) 205-223.

[3] Y. Gelbstein, Z. Dashevsky, M.P. Dariel, High performance n-type PbTe-based materials for thermoelectric applications, Physica B Condens. Matter. 363 (2005) 196-205.

[4] K. Ahn, K. Biswas, J. He, I. Chung, V. Dravid, M.G. Kanatzidis, Enhanced thermoelectric properties of p-type nanostructured PbTe-MTe $(\mathrm{M}=\mathrm{Cd}, \mathrm{Hg})$ materials, Energy Environ. Sci. 6 (2013) 1529.

[5] S. Sano, H. Mizukami, H. Kaibe, Development of High-Efficiency Thermoelectric Power Generation System, Komatsu Tech. Rep. 49 (2003) 2026.

[6] A.I. Casian, I.I. Sanduleac, ORGANIC THERMOELECTRIC MATERIALS : NEW OPPORTUNITIES, J. Thermoelectr. 3 (2013) 11-20.

[7] E. a. Perez-Albuerne, Highly Conducting Ion-Radical Salts of Tetrathiotetracene, J. Chem. Phys. 55 (1971) 1547.

[8] J. Wüsten, K. Potje-Kamloth, Organic thermogenerators for energy autarkic systems on flexible substrates, J. Phys. D. Appl. Phys. 41 (2008) 135113.

[9] A. Casian, Violation of the Wiedemann-Franz law in quasi-one-dimensional organic crystals, Phys. Rev. B. 81 (2010) 155415.

[10] D. Knipp, R. a. Street, a. Völkel, J. Ho, Pentacene thin film transistors on inorganic dielectrics: Morphology, structural properties, and electronic transport, J. Appl. Phys. 93 (2003) 347.

[11] W.S. Hu, Y.T. Tao, Y.J. Hsu, D.H. Wei, Y.S. Wu, Molecular orientation of evaporated pentacene films on gold: alignment effect of self-assembled monolayer., Langmuir. 21 (2005) 2260-2266.

[12] N. Koch, a. Elschner, J. Schwartz, a. Kahn, Organic molecular films on gold versus conducting polymer: Influence of injection barrier height and morphology on current-voltage characteristics, Appl. Phys. Lett. 82 (2003) 2281.

[13] H. Inokuchi, M. Kochi, Y. Harada, Electrical Properties of Tetrathiotetracene, Bull. Chem. Soc. Jpn. 40 (1967) 2695-2696. 
[14] J. Webb, D. Williams, Hopping conduction in thin films of tetrathiotetracene, J. Phys. C Solid State Phys. 12 (1979) 3173-3183.

[15] E.A. Silinsh, L. Taure, Some electrophysical and photoelectrical properties of thin layers systems of tetrathiotetracene, Izv.AN Latv. Ser. Fiz. Techn.Nauk. 1 (1970) 18-27.

[16] D. Balode, E.A. Silinsh, Conductivity mechanisms and refined energy level spectrm of tetrathiotetracene, Izv.AN Latv. Ser. Fiz. Techn.Nauk. 1 (1978) 3545 .

[17] B. Fiedler, E. Rojo-Wiechel, J. Klassen, J. Simon, J. Beck, M. Sokolowski, Ordered structures of two sulfur containing donor molecules on the $\mathrm{Au}(111)$ surface, Surf. Sci. 606 (2012) 1855-1863.

[18] E.A. Perez-Albuerne, Synthesis of chalcogenated polyacenes, (Eastman Kodak Co.), U.S. Patent $372341719730327,1973$.

[19] G. Juska, K. Arlauskas, M. Viliunas, J. Kocka, Extraction current transients: new method of study of charge transport in microcrystalline silicon, Phys. Rev. Lett. 84 (2000) 4946-4949.

[20] G. Juška, K. Arlauskas, M. Viliu, K. Genevic, G. Jus, H. Stubb, Charge transport in p-conjugated polymers from extraction current transients, Phys. Rev. B. 62 (2000) 235-238.

[21] B. Hilti, C.W. Mayer, Electrical Properties of the Organic Metallic Compound bis (Tetrathiotetracene)-Triiodide, (TTT)2I3, Helv. Chim. Acta. 61 (1978) 501-511.

[22] Y. Matsunaga, Some Molecular Complexes of Tetrathiotetracene Exhibiting Low Electrical Resistivities, J. Chem. Phys. 42 (1965) 2248. 